\title{
Time-dependent associations between presentation-based infections and subsequent risk of childhood immune cell malignancies
}

TienYu Owen Yang MD DPhil; Kuan-Ying Arthur Huang MD DPhil; Mei-Huei Chen MD PhD; PauChung Chen MD PhD; Wan-Ting Huang MD

1 Nuffield Department of Population Health, University of Oxford, Richard Doll Building, Old Road Campus, Oxford OX3 7LF, UK

2 Division of Pediatric Infectious Diseases, Department of Pediatrics, Chang Gung Memorial Hospital and School of Medicine, Chang Gung University, 333, Taiwan, Taoyuan City, Guishan District

3 Institute of Population Health Sciences, Taiwan National Health Research Institutes, No. 35, Keyan Road, Zhunan Township, Miaoli County, Taiwan 350

4 Institute of Occupational Medicine and Industrial Hygiene, National Taiwan University College of Public Health; Department of Public Health, National Taiwan University College of Public Health; Department of Environmental and Occupational Medicine, National Taiwan University College of Medicine and Hospital, No. 17, Xuzhou Road, Zhongzheng District, Taipei City, Taiwan 100

5 Taiwan Centers for Disease Control, No. 6, Linsen South Road, Zhongzheng District, Taipei City, Taiwan 100

Running title: Infection and risk of childhood leukaemias and lymphomas

Word count: highlights 68, abstract 249, manuscript 2744

Correspondence to:

Dr TienYu Owen Yang, Nuffield Department of Population Health, University of Oxford 
$08 / 07 / 2020$

Richard Doll Building, Old Road Campus, Oxford OX3 7LF, UK

owen.yang@ndph.ox.ac.uk

All authors declared no conflict of interest.

The study was funded by the University of Oxford Nuffield Department of Population Health PumpPriming Award (H6D00410). The study protocol was reviewed and approved by the Institutional Review Board (IRB) at National Taiwan University Hospital (201705028RINA), Chang Gung Memorial Hospital (105-4832C), and Taiwan Centers for Disease Control (105205). The research was performed in accordance with the relevant guidelines and regulations. The IRBs waived the requirement for obtaining individual informed consent because effectively only anonymized datasets were used in this study. Databases in this study should be accessed directly via Taiwan Ministry of Health and Welfare.

Ideally colour should be used to the figures, but we may provide grey-scale figures if necessary. 
$08 / 07 / 2020$

\section{HIGHTLIGHTS}

Associations between individual infections and childhood immune cell malignancies are reported.

Some may be due to infection caused by malignancies already active prior to diagnosis.

Guided by unexplained lymph nodes, we found malignancies could be active years before diagnosis.

We also found increase in multiple infection presentations prior to malignancy diagnosis.

In all, most apparent associations could not be largely attributed to infection as a cause of malignancy. 
$08 / 07 / 2020$

\begin{abstract}
Background

Infection is hypothesised as a contributory cause of childhood immune cell malignancies. Although some have reported associations between individual infections and immune cell malignancies, some could be spurious due to infections caused by malignancies that were already active prior to diagnosis.
\end{abstract}

\title{
Methods
}

Identified from Taiwan Cancer Registry, 3000 children with four commonest immune cell malignancies diagnosed during 2001-2015 at age 1-20 years were identified and matched with 1:10 controls. Using logistic regression, we estimated the time-specific case-versus-control odds ratios of seven common infection presentations in their health records. We also compared recorded unexplained lymph nodes between cases and controls to explore for how long malignancy may be active prior to diagnosis.

\section{Results}

Unexplained lymph nodes were increasingly recorded months before the diagnosis of childhood leukaemias and years before the diagnosis of childhood lymphomas. When using $\mathrm{p}<0.01$ as a guide, large case-control differences in infection records were found mostly within $0-2$ months prior to the diagnosis (15 out of 28 comparisons). Changes in odds ratios within 3-35 months (2 out of 28 comparisons) and case-control differences beyond 36+ months prior to diagnosis ( 7 out of 28 comparisons) was relatively small ( $\sim 10 \%$ difference in leukaemias). Statistical power varied according to incidence of malignancy, incidence of infection records, and the age distribution.

\section{Conclusion}

Immune cell malignancies were likely to be active some time before the diagnosis. Previous studies using conventional population-based methods may not be able to distinguish any small causal link between infection and immune cell malignancies from spurious associations. 
$08 / 07 / 2020$

Keywords: infection; childhood leukaemia; lymphomas; reverse causation; enterovirus; tooth cavity; periodontic disease 


\section{BACKGROUND}

Infection has been long hypothesised as a contributing factor in the oncogenesis of childhood leukaemias and lymphomas (1-5), and some studies have reported links between individual presentations of infection and subsequent risk of individual immune cell malignancies (6-10). However, it is known that children with immune cell malignancies develop multiple presentations prior to malignancy diagnosis, and some presentations could either mimic infection or be caused by infection (11). An apparent association could be spurious due to infection as a presentation of immune cell malignancies.

Investigation into time-dependent associations across various infections and presentations simultaneous may aid assessment of these findings. A multiple-presentation association between infection and immune cell malignancy, especially those associations that become stronger near the time of malignancy diagnosis, is likely to be caused by the underlying active malignancy prior to diagnosis. Here we present time-dependent associations across seven commonest infection presentations in Taiwan prior to the diagnosis of four childhood immune cell malignancies.

\section{METHODS}

From the histologically confirmed Taiwan Cancer Registry $(12,13)$, we identified children with acute lymphoid leukaemia, acute myeloid leukaemia, non-Hodgkin lymphoma, and Hodgkin lymphoma diagnosed between age 1 and 20 years in 2001-2015 using International Classification of Childhood Cancer, Third Edition (ICCC-3).(14) Using the data linkage framework hosted by the Department of Health and Welfare, the information in the Taiwan Cancer Registry could be linked to the healthcare database in Taiwan, the Taiwan National Health Insurance Research Databases, which had covered $>99 \%$ of the Taiwanese population ( $\sim 23$ millions). For each child, up to 10 children of the same sex, year and month of birth, county-level region, and 4 levels of urbanisation (15) were selected from the healthcare database as population controls. All controls contributed person-months at the same age and during the same time as the matched cases, from the month of birth or January 2000, whichever 
the later, to the month of malignancy diagnosis. The study protocol was reviewed and approved by the Institutional Review Board (IRB) at National Taiwan University Hospital (201705028RINA), Chang Gung Memorial Hospital (105-4832C), and Taiwan Centers for Disease Control (105205).

Presentation-based infectious conditions recorded within the contributed person-months were also extracted, including commonest respiratory, gastrointestinal, mucocutaneous, and dental conditions, according to an algorithm based on International Classification of Diseases, $9^{\text {th }}$ revision. (ICD-9 see Appendix A for the extraction algorithm). Because Taiwanese children access healthcare very often even for mild conditions, this algorithm was designed to prioritise more specific presentations when a specific and non-specific presentations were both recorded in the person-month.

We also extracted the records of lymph nodes of unspecified cause based on ICD-9 codes (Appendix A). These were records of lymph nodes without specified cause, and so they were not overt acute lymphadenitis or infectious mononucleosis. However, they had to be seen by doctors in order to be recorded, and so were likely to be under-reported in the healthcare system. Nevertheless, this lymph nodes of no apparent cause recorded by doctors, when compared between cases and controls, provided information as to a conservative time frame when underlying malignancy could have been active before diagnosis.

Statistical analysis and data use considerations

This study was one of the earliest studies with data linkage of the healthcare records for the entire Taiwanese childhood population, including those who have had malignancies. The study analysis was therefore designed taking into consideration of complying with data use agreement, minimising unnecessary risk of inappropriate data use that may re-identify individuals, and optimising cost and time management. Updated regulation and guidance, and accessing the data, can be found on the website of the Taiwan Ministry of Health and welfare (https://www.mohw.gov.tw).

For each of the four types of immune cell malignancies and each of the seven type of infectious presentations and for lymph nodes of unspecified cause, we estimated the case-versus-control odds ratios (OR) of having an eventful person-month (i.e. having a record of a type-specific presentations 
during that month) at each 3-month interval, counting backwards from the time of diagnosis $(0-2,3-$ $5, \ldots, 32-35$, and $36+$ months), and used three summary statistics according to the time periods prior to malignancy diagnosis: (1) case-versus-control OR for having an eventful person-month during 0-2 months prior to malignancy diagnosis, (2) the upward or downward linear trend of case-versus-control ORs during 3-35 months prior to the malignancy diagnosis, and (3) the case-versus-control OR 36+ months prior to the malignancy diagnosis. Because the cases and controls were matched by sex, time, age, and region with a nearly fixed case-control ratio at 1:10, each control contributed to the same amount of person-months as the matched case, and in the case-control ratio of person-months was fixed at 1:10 in each case-control stratum. In this sample where characteristics were matched and balanced between cases and controls, OR at each time period prior to diagnosis were estimated at the person-month level using logistic regression. In practice, the ORs of having an eventful month (cases versus controls) were estimated by 3 -monthly time periods prior to diagnosis $(0-2,3-5,6-8, \ldots, 32-35$, $36+$ months prior to diagnosis) using an interaction term between case-control identifier and 13 time periods, and the case-control odds ratios and standard errors at each time period were obtained by reassigning baseline categories for each time period of interest (using logit and lincom in STATA). Once the estimates and standard errors of case-versus-control ORs for all 3-month time periods were obtained, the linear trend of odds ratio from the $3^{\text {rd }}$ to the $35^{\text {th }}$ month prior to diagnosis was then estimated using meta-regression (metareg in STATA), interpreted as change in OR for every 3 months nearer to diagnosis.

With multiple time periods, events, and malignancy types, there was uncertain chance of false positive and false negative findings. We presented estimates, $95 \%$ confidence intervals, and crude $\mathrm{p}$ value at three levels $(<0.01,<0.001$, and $<0.001)$ as guide, but interpreted robustness of findings based on the strength of significance and general pattern of findings.

To avoid risk of individuals to be re-identified, we presented the actual total numbers of cases and controls and the distribution of person-months by age to the nearest 10 s, and estimated the distribution of age at diagnosis based on published Taiwan Cancer Registry data in 2001-2015, We also estimated 
the event numbers using inverse variance weight of odds ratios. The observed trend was smoothed using a cubic function (metareg in STATA).

\section{RESULTS}

During 2001-2015, we identified $\sim 1470$ children with acute lymphoid leukaemia, $\sim 630$ children with acute myeloid leukaemia, $\sim 600$ children with non-Hodgkin lymphoma, and $\sim 370$ children with Hodgkin lymphoma diagnosed between age 1 and 20 years. In Taiwan the peak age of acute childhood lymphoid leukaemias was in early childhood, and the peak ages of other immune cell malignancies were in adolescence (Appendix B). With few exceptions, a fixed number of 10 controls were matched for almost all cases. The number of person-months by age and estimated eventful person-months are also shown in Appendix B. Note that mild respiratory infections (i.e. other acute respiratory infections) was very commonly recorded in Taiwan. For example, each child with acute lymphoid leukaemia contributed 60 months of person-months on average prior to leukaemia diagnosis, among which approximately 25 months were recorded with other acute respiratory infections only. Person-months with other infection presentations accounted for addition 11 months on average (Appendix B).

Unexplained lymph nodes were recorded prior to immune cell malignancy diagnosis

For children with acute lymphoid leukaemia and acute myeloid leukaemia, we found that lymph nodes of unspecified cause were not recorded more often than matched controls until 0-2 months prior to the diagnosis, when the amount of records increased sharply (OR $=47,95 \%$ CI $29-76, p<0.0001$ for acute lymphoid leukaemia; $\mathrm{OR}=17,7-44, \mathrm{p}<0.0001$ for acute myeloid leukaemia, Figure 1). There was weak evidence for a higher number of lymph node records $35+$ months prior to the diagnosis of acute lymphoid leukaemia compared to controls $(\mathrm{OR}=1.6,1.0-2.5, \mathrm{p}=0.04)$. For non-Hodgkin lymphoma and Hodgkin lymphoma, there were also sharp increases 0-2 months prior to the diagnosis 
$(\mathrm{OR}=110,57-211$ for non-Hodgkin lymphoma, $\mathrm{p}<0.0001$; $\mathrm{OR}=1309,183-9378$, for Hodgkin lymphoma; $\mathrm{p}<0.0001$ ), but the lymph node records had been building up in the period 3-35 months prior to these diagnoses (OR increase by 0.28 per 3 months for non-Hodgkin lymphoma, $\mathrm{p}$ for linear trend $=0.001$; OR increase by 0.31 per 3 months for Hodgkin lymphoma, $\mathrm{p}$ for linear trend $<0.0001$, Figure 1). In Hodgkin lymphoma, substantial case-control difference was also found even more than three years prior to the diagnosis $(\mathrm{OR}=2.61 .5-4.5, \mathrm{p}=0.001)$. All estimates and $\mathrm{p}$ values are listed in Appendix C.

Infection presentations recorded 0-2 months prior to malignancy

Compared to controls, there were more records in multiple infection presentations in children within 0-2 months prior to malignancy diagnosis, evident in 15 out of 28 pairs of infection-malignancy associations when $\mathrm{p}<0.01$ was used as a guide (Figure 2, and see Appendix $\mathrm{C}$ for estimates). Specifically, for all four immune cell malignancies there was evidence of more records in pneumonia and influenza, other acute respiratory infections, and acute gastrointestinal infections; for acute lymphoid leukaemia there were more records in periodontal diseases; for acute myeloid leukaemia there were more records in non-enterovirus viral exanthems, periodontal diseases, and dental caries (Figure 2 and Appendix C).

Infection presentation recorded in the period 3-35 months prior to malignancy diagnosis

In the period of 3-35 months prior to the diagnosis of acute myeloid lymphoma, the OR of pneumonia and influenza had seemed to increase in period of 3-35 months period to the diagnosis of acute myeloid leukaemia ( $\mathrm{p}$ for linear trend over 33 months $=0.0003$ ), and the smoothed observed trend (Figure 2) suggested a continuous increase over this time period. We did not find any other increasing trend in other infection presentations or in other malignancies. However, the p value for linear trend suggested that the ORs of enterovirus exanthems had been declined gradually in the period of 3-35 
months prior to the diagnosis of acute lymphoid leukaemia ( $\mathrm{p}$ for linear trend over 33 months $=0.002$, Figure 2 and Appendix C). When we investigate separately for two main subtypes of enterovirus exanthems with different virologic profiles,(16) the downward linear trends of OR seemed to be restricted to herpangina but not seen in hand, foot, and mouth disease (Figure 3 and Appendix C)

Infection presentations 36 or more months prior to malignancy diagnosis

Beyond three years prior to malignancy diagnosis, the statistical evidence suggesting a case-control difference was scattered, and the ORs were small in most occasions. Compared to matched controls, there was evidence suggesting more records of acute gastroenteritis $36+$ months prior to the diagnosis of acute lymphoid leukaemia $(\mathrm{OR}=1.11,1.03-1.19, \mathrm{p}=0.004)$; more records in pneumonia and influenza $(\mathrm{OR}=1.12,1.03-1.21, \mathrm{p}=0.007)$ and periodontal diseases $(\mathrm{OR}=1.13,1.04-1.23, \mathrm{p}=0.005)$ prior the diagnosis of acute myeloid leukaemia; more records in pneumonia and influenza $(\mathrm{OR}=1.14$, 1.06-1.23, $\mathrm{p}=0.0008)$ but fewer records in other acute respiratory infections $(\mathrm{OR}=0.95,0.93-0.97$, $\mathrm{p}<0.0001)$ prior to the diagnosis of non-Hodgkin lymphoma; fewer records in pneumonia and influenza $(\mathrm{OR}=0.79,0.71-0.88, \mathrm{p}<0.0001)$ and in dental caries $(\mathrm{OR}=1.18,1.12-1.25, \mathrm{p}<0.0001)$ prior to Hodgkin lymphoma.

\section{DISCUSSION}

Using a time-dependent approach with the largest and prospectively collected healthcare data, we examined as many infection presentations and as many immune cell malignancies as feasible, so that the it was possible to assess whether any time-specific associations was common across presentations and immune cell malignancies. In an investigation into infectious causes of immune cell malignancies, these comparisons added information with which the pattern a spurious, non-causal association could be better revealed. 
Spurious associations may occur during the period when an active malignancy already existed prior to malignancy diagnosis. Children whose malignancy was active long before diagnosis could start to have various infectious and non-infectious symptoms, and a spurious association between infection and following diagnosis of immune cell malignancies may appear even if infection was not a contributory cause of malignancy. We used records of lymph nodes as a putative guide for the time periods when an active malignancy already existed prior to diagnosis. Our results suggested this active phase could be traced at least months prior to leukaemia diagnosis, and years prior to lymphoma diagnosis. Therefore, most apparent time-dependent associations between infection presentations and immune cell malignancies during this time period cannot be entirely attributed to infection as a cause of immune cell malignancies. The multiple presentations in a few months prior to the diagnosis of childhood leukaemias were largely consistent with our report on specific and nonspecific symptoms prior to childhood leukaemias of a smaller sample (11), and these infection-like presentations could not be distinguished from malignancy symptoms. The increasing rate of pneumonia and influenza in the 3-35 months prior to acute myeloid leukaemia has been suspected before $(6,10)$, but we finding of linear trends for change in case-versus-control ORs demonstrated an accelerated increase during this time period, a pattern more suggestive of infection as a marker of underlying malignancy burden instead of as an aetiology of leukaemia. The reduction in rate of enterovirus exanthems prior to the diagnosis of acute lymphoid leukaemia has also been suspected before in Taiwan but with little information on time specificity (9). Here we demonstrated this reduction seemed to be restricted to herpangina, and largely confined to the short periods prior to leukaemia diagnosis. This pattern may also be more consistent with a marker of underlying malignancy.

Spurious associations could also occur due to chance when comparisons were made either selectively or non-selectively, and so the scattered associations of a longer term in our study are difficult to interpret. For example, the associations with strongest statistical significance $(p<0.001)$ were limited to lymphomas, for which lymph nodes could be found more common in cases than controls many years prior to diagnosis, and so it was difficult to ascertain whether infection predated malignancy. 
There was no large case-control difference in lymph node of unspecified cause recorded in the longer period prior to the diagnosis of acute leukaemias, and so the differences in infection presentations during a longer period of time prior to diagnosis may be supportive of infection as a contributary cause. There were a few potential differences in infection presentations identified $35+$ months prior to the diagnosis of leukaemias compared to matched controls at a weaker level of statistical significance $(\mathrm{p}<0.01$ but $\mathrm{p}>0.001)$ and small estimated difference (OR near 1.1 in all three associations), including more acute gastroenteritis recorded more than three years prior to diagnosis of acute lymphoid leukaemia, and more pneumonia and influenza or more periodontal diseases more than three years prior to the diagnosis of acute myeloid leukaemia. Some of these apparent associations, if reproducible, could be driven by causal mechanisms, but the small odds ratios may suggest that the direct mechanisms were poorly captured through these infection records.

Despite the effort to include all cases in Taiwan across 15 years in the $21^{\text {st }}$ century and the high medical access in Taiwan that allows maximal recording of infectious events, the study was still mainly limited by the amount of cases and person-months. Acute lymphoid leukaemia was the commonest childhood malignancy, but the age of diagnosis was relatively young, resulting in a relatively small proportion of contributed person-time more than three years prior to malignancy diagnosis. For example, the statistically weak but relatively non-trivial $(\mathrm{OR}=1.6$, Figure 1$)$ increase in lymph nodes of unspecified cause recorded $35+$ months prior to the diagnosis of acute lymphoid leukaemia could be due to limited sample size, due to poorly characterised events, or due to chance. The data were lack of biomolecular details of infection and of malignancies that may refine our hypothesis on an infectious cause of malignancy, but again any more details will require sufficient case numbers to investigate. Even with the characteristics that seem available, stratification of analysis for further heterogeneity by infection or malignancy subtypes, or by other characteristics would require a larger case number for meaningful comparisons. Estimation of absolute risks of malignancy associated with infections would have not been meaningful without investigation into these heterogeneities. Extending analyses into rarer infection or malignancy types, or other specified 
causes of lymph node involvement such as infectious mononucleosis, would also require a larger case number.

To our knowledge this is the first report to quantitatively demonstrate and compare time-dependent associations across infection presentations and immune cell malignancies in children. In all, it was common to find an apparent association between infection presentations and subsequent risk of a malignancy diagnosis, but the time-dependent patterns suggested a strong component of non-causality with which any causal association could be overwhelmingly masked.

\section{ACKWOLEDGEMENT}

We thank Yu-Chan Chen and Ya-Chi Chang for their assistance in the application for data linkage and ethics approval.

\section{Ethics approval, funding, and data availability}

The study was funded by the University of Oxford Nuffield Department of Population Health PumpPriming Award (H6D00410). The study protocol was reviewed and approved by the Institutional Review Board (IRB) at National Taiwan University Hospital (201705028RINA), Chang Gung Memorial Hospital (105-4832C), and Taiwan Centers for Disease Control (105205). The research was performed in accordance with the relevant guidelines and regulations. The IRBs waived the requirement for obtaining individual informed consent because effectively only anonymized datasets 
were used in this study. Databases in this study should be accessed directly via Taiwan Ministry of Health and Welfare.

Author contributions and conflict of interest

TOY and WTH conceived and designed the study. TOY, MHC, PCC, and WTH designed the main data framework. TOY was the primary analyst and drafted the manuscript. WTH contributed to the analysis. All authors have critically interpreted the findings, contributed to writing, and agreed to the submission of manuscript. The corresponding author had full access to all the data in the study and had final responsibility for the decision to submit for publication. All authors declared no conflict of interest. 


\section{REFERENCE}

1. Greaves M. A causal mechanism for childhood acute lymphoblastic leukaemia. Nature reviews Cancer. 2018;18:471-84.

2. Kinlen L. Childhood leukaemia, nuclear sites, and population mixing. Br J Cancer. 2011;104:12-8.

3. Greaves M. Infection, immune responses and the aetiology of childhood leukaemia. Nature reviews Cancer. 2006;6:193-203.

4. Rudant J, Lightfoot T, Urayama KY, Petridou E, Dockerty JD, Magnani C, et al. Childhood acute lymphoblastic leukemia and indicators of early immune stimulation: a Childhood Leukemia International Consortium study. Am J Epidemiol. 2015;181:549-62.

5. Marcotte EL, Ritz B, Cockburn M, Yu F, Heck JE. Exposure to infections and risk of leukemia in young children. Cancer Epidemiol Biomarkers Prev. 2014;23:1195-203.

6. Chang JS, Tsai CR, Tsai YW, Wiemels JL. Medically diagnosed infections and risk of childhood leukaemia: a population-based case-control study. Int J Epidemiol. 2012;41:1050-9.

7. Vestergaard TR, Rostgaard K, Grau K, Schmiegelow K, Hjalgrim H. Hospitalisation for infection prior to diagnosis of acute lymphoblastic leukaemia in children. Pediatric blood \& cancer. 2013;60:428-32.

8. Crouch S, Lightfoot T, Simpson J, Smith A, Ansell P, Roman E. Infectious illness in children subsequently diagnosed with acute lymphoblastic leukemia: modeling the trends from birth to diagnosis. American Journal of Epidemiology. 2012;176:402-8.

9. Lin JN, Lin CL, Lin MC, Lai CH, Lin HH, Yang CH, et al. Risk of leukaemia in children infected with enterovirus: a nationwide, retrospective, population-based, Taiwanese-registry, cohort study. The Lancet Oncology. 2015.

10. Sogaard KK, Farkas DK, Sorensen HT. Pneumonia diagnosis in childhood and incidence of leukaemia, lymphoma and brain cancer: a Danish nationwide cohort study. BMJ Open.

2017;7:e019860.

11. Yang TO, Liu YL, Huang WT, Chen MH, Chen PC. Specific and Non-specific Clinical Presentations in the Year Before the Diagnosis of Childhood Leukaemia. Pediatr Blood Cancer. 2016;63:1387-93.

12. Chiang CJ, You SL, Chen CJ, Yang YW, Lo WC, Lai MS. Quality assessment and improvement of nationwide cancer registration system in Taiwan: a review. Jpn J Clin Oncol. 2015;45:291-6. 13. Liu YL, Lo WC, Chiang CJ, Yang YW, Lu MY, Hsu WM, et al. Incidence of cancer in children aged 0-14 years in Taiwan, 1996-2010. Cancer Epidemiol. 2015;39:21-8.

14. Steliarova-Foucher E, Stiller C, Lacour B, Kaatsch P. International Classification of Childhood Cancer, third edition. Cancer. 2005;103:1457-67.

15. Yang TYO, Huang W-T, Chen M-H, Chen P-C. Seasonal synchrony in incidences of common infectious diseases in early childhood among neighbouring regions. International Journal of Infectious Diseases. 2014;28:214.

16. Yang TO, Huang KA, Chen MH, Chen PC, Huang WT. Comparison of Nonpolio Enteroviruses in Children With Herpangina and With Hand, Foot and Mouth Disease in Taiwan. The Pediatric infectious disease journal. 2019. 
Figure 1. Relative numbers of records of lymph nodes of unspecified cause by time to the diagnosis of four childhood immune cell malignancies compared to time-appropriate matched controls.

Asterisks shows $\mathrm{p}$ values at $<0.01(*),<0.001(* *)$, and $<0.0001(* * *)$ for each time-specific analysis, including case-versuscontrol odds ratios (ORs) 0-2 months prior to diagnosis (in orange asterisks, dots showing point estimates), linear change of case-versus-control OR during 3-35 months prior to diagnosis (in red, shadows showing 95\% confidence intervals of smoothed observed trend), and case-versus-control ORs $35+$ prior to diagnosis (in green, bar showing $95 \%$ confidence intervals).
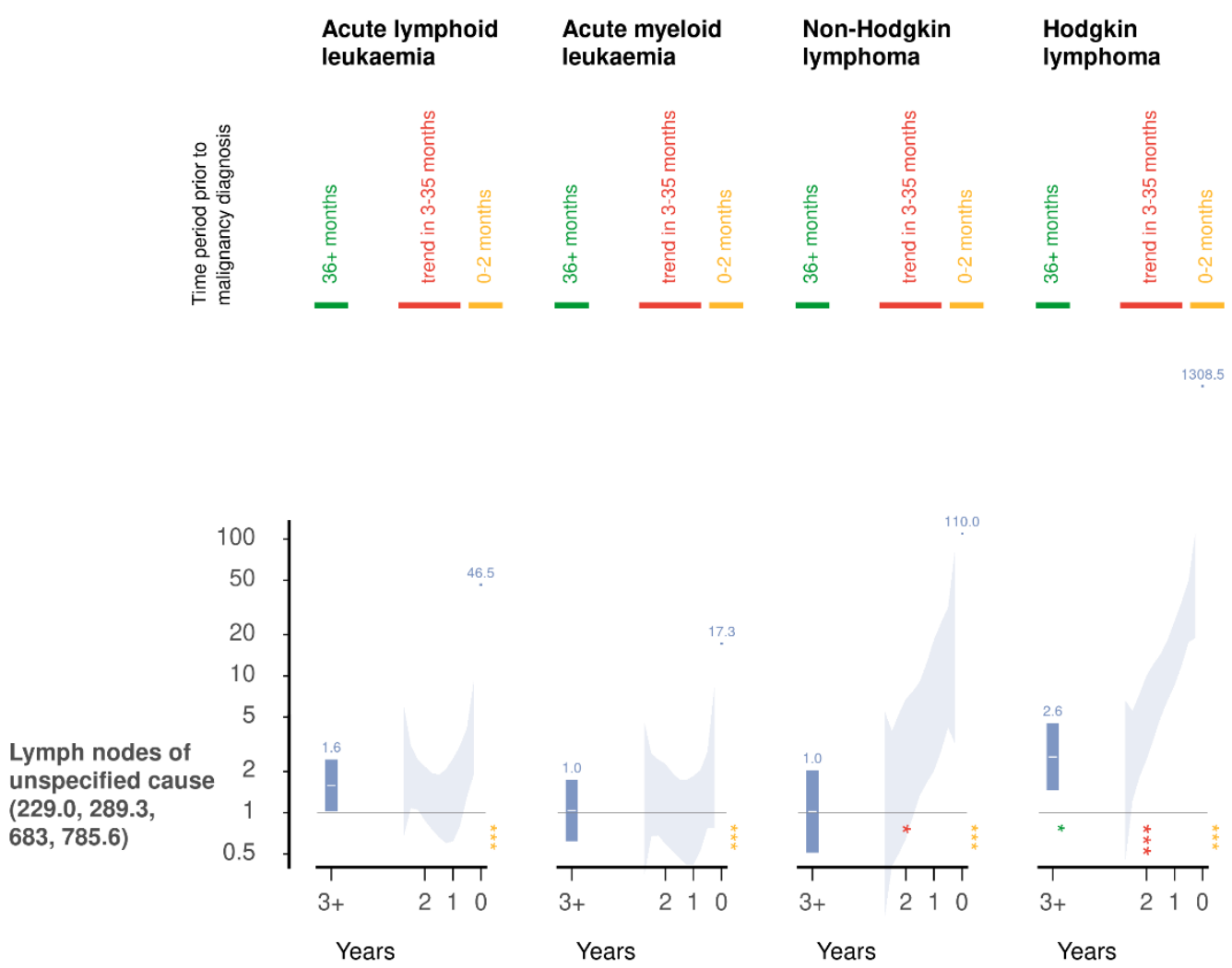
Figure 2. Relative numbers of records of eight presentation-based infections by time to the diagnosis of four childhood immune cell malignancies compared to time-appropriate matched controls.

Asterisks shows $\mathrm{p}$ values at $<0.01(*),<0.001(* *)$, and $<0.0001(* * *)$ for each time-specific analysis, including case-versuscontrol odds ratios (ORs) 0-2 months prior to diagnosis (in orange asterisks, dots showing point estimates), linear change of case-versus-control OR during 3-35 months prior to diagnosis (in red, shadows showing 95\% confidence intervals of smoothed observed trend), and case-versus-control ORs 35+ prior to diagnosis (in green, bar showing $95 \%$ confidence intervals).

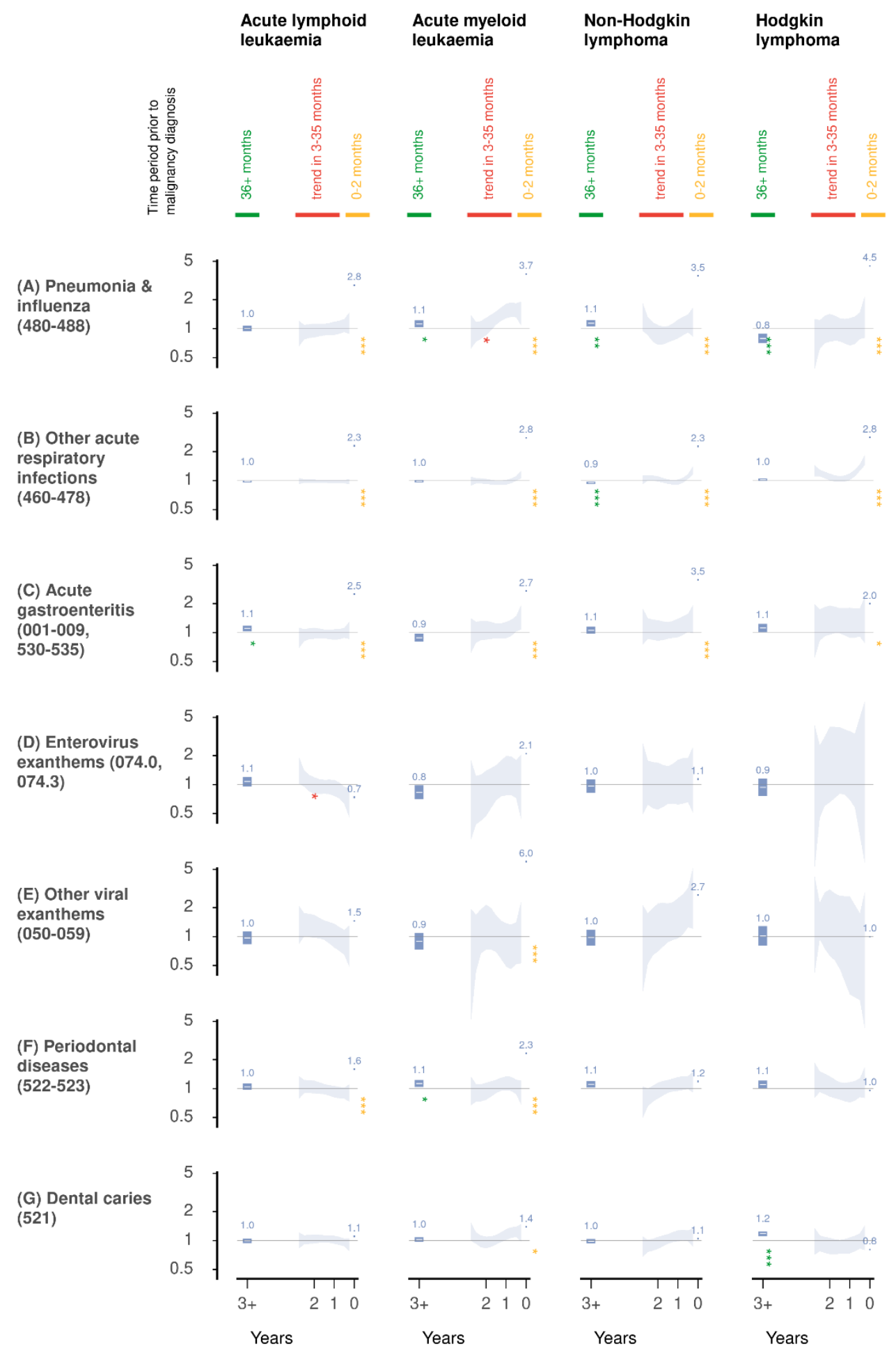


Figure 3. Relative numbers of records of enterovirus exanthem subtypes by time to the diagnosis of acute lymphoid leukaemia compared to time-appropriate matched controls.

Asterisks shows p values at $<0.01(*),<0.001(* *)$, and $<0.0001(* * *)$ for each time-specific analysis, including case-versuscontrol odds ratios (ORs) 0-2 months prior to diagnosis (in orange asterisks, dots showing point estimates), linear change of case-versus-control OR during 3-35 months prior to diagnosis (in red, shadows showing 95\% confidence intervals of smoothed observed trend), and case-versus-control ORs 35+ prior to diagnosis (in green, bar showing 95\% confidence intervals).

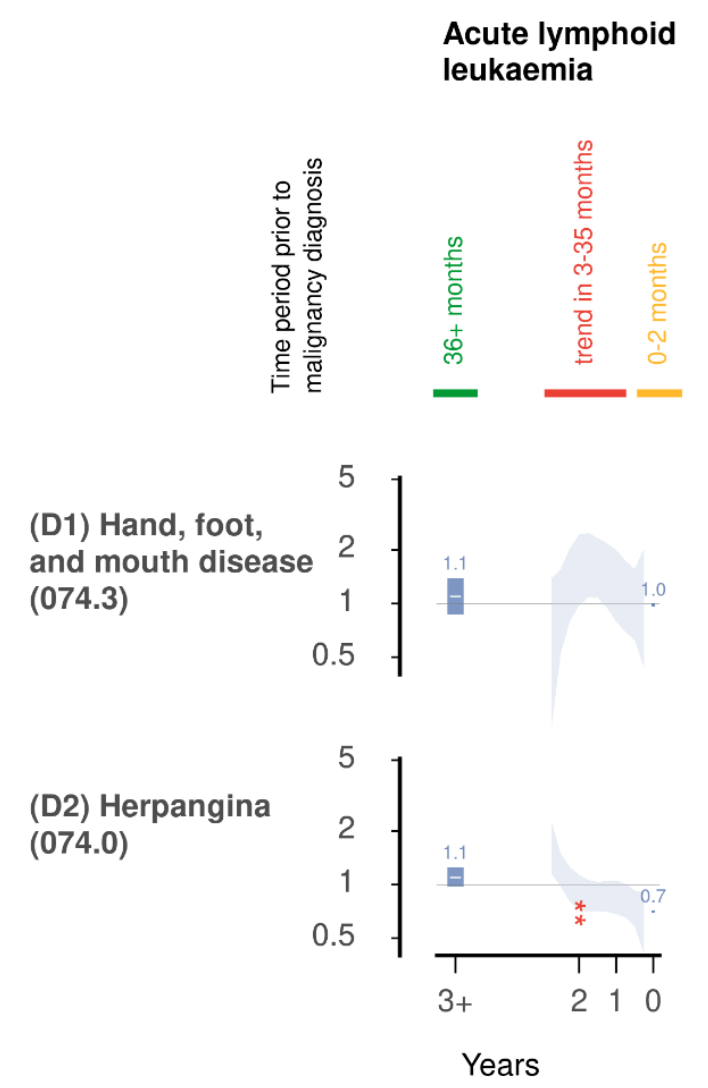

